\title{
Reconstrucción auricular: experiencia de 24 años
}

\section{Auricular reconstruction: 24 year experience}

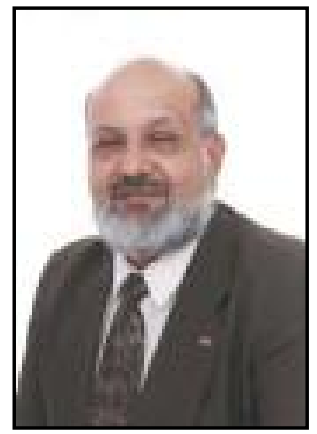

Mantilla Martín, A. I.

Mantilla Martín, A. I.*

Abstract

La oreja, debido a su situación expuesta en la cabeza, es un órgano susceptible de sufrir múltiples traumas e incluso pérdida; así mismo, es asiento de neoplasias malignas, carcinomas y melanomas que igualmente finalizan en amputación. Fuera de estas razones, la ausencia congénita del pabellón auricular es la segunda malformación facial de origen congénito más frecuente después del labio y paladar fisurados. Es común por tanto para el cirujano plástico verse frente a pacientes que consultan solicitando la solución a su deformidad.

El presente artículo trata de señalar el camino a seguir dividido en cuatro etapas de acuerdo a los principios definidos por el Dr. Radford Tanzer y perfeccionados a través de los años por el Dr. Burt Brent, e ilustra el manejo de la reconstrucción en casos de microtia, amputación auricular y el uso de colgajos de fascia temporal en los casos en que la piel de la región mastoidea ha sido comprometida y no es posible usarla como elemento primario para la reconstrucción auricular.

\section{Palabras clave Microtia. Reconstrucción auricular.}

Due to its very exposed situation on both sides of the head, the auricle is very susceptible to suffer severe traumas, that will at the end cause its amputation; it's too one of the most common sites to present malignant tumors, carcinoma and melanoma, that will also end in the surgical amputation of the ear. Congenital absence of the ear, microtia, is the second most common facial anomaly after cleft lip and palate. Not infrequently plastic surgeon confronts with patients seeking an answer for correction of their deformities.

The present article tries to show a four steps method, according to the principles described first by Dr. Radford Tanzer and later improved by Dr. Burt Brent and illustrates how to manage the auricular reconstruction in cases of congenital microtia or traumatic loss of the auricle, as well as the use of the temporal fascia flap as a way to provide skin coverage, when the skin of the mastoid area has been compromised and it is not useful for auricular reconstruction.

$\begin{array}{ll}\text { Key words } & \text { Microtia. Auricular reconstruction. } \\ \text { Numeral Gode } & 2545,2447\end{array}$




\section{Introducción}

Debido a su situación expuesta a ambos lados de la cabeza, las orejas son susceptibles de sufrir múltiples agresiones que pueden producir como resultado la pérdida traumática del pabellón auricular. También son uno de los asientos preferidos de las neoplasias malignas y en consecuencia se ven sujetas a la resección parcial o total por parte de los cirujanos. Por último, pero no menos importante, la ausencia congénita del pabellón auricular, que es la segunda malformación facial más frecuente después del labio y el paladar fisurados, hace que el capítulo de la reconstrucción auricular en Cirugía Plástica sea una etapa obligada, aunque no muy frecuentada, por todos los cirujanos plásticos, pues la reconstrucción de la oreja es uno de los retos más formidables que puede enfrentar un especialista.

En la primera edición de su libro "Arte y Principios de la Cirugía Plástica", el profesor Sir Harold Gillies, en el capítulo correspondiente a la reconstrucción auricular, escribía: "Tanto Silla como Caribdis acechan el camino que sigue el cirujano plástico que intenta reconstruir una oreja; demasiadas veces el cirujano termina elaborando un pegote o una serie de pegotes muy bajos en la cabeza y con proyección insuficiente. Algo que damos en llamar una oreja, pero que no es, ni se parece a una oreja”. Mas adelante continua el autor, "Es el natural deseo de las personas que las orejas sean suyas y que no se caigan al menor movimiento de la cabeza o al quitarse el sombrero". También relata en su libro como un marino danés, que había perdido su oreja en una explosión y tenía una muy imperfecta reconstrucción auricular decía: "Mi nueva oreja no es buena, pero da una sensación de balance y simetría a mi cabeza. La ausencia de la oreja me daba una sensación de mutilación, la misma que sentiría si tuviese un brazo o una pierna amputados" (1).

En estos tres relatos, se resumen las dificultades que involucran una reconstrucción auricular; por un lado lograr tallar en cartílago un molde adecuado que pueda imitar la forma de una oreja y proveerlo luego de una cubierta cutánea adecuada que refleje los detalles de la nueva oreja; ésta debe estar adecuadamente colocada en la cabeza de manera que se vea natural y simétrica con la oreja sana. Así mismo, debe ser permanente, capaz de tolerar las circunstancias de la vida diaria sin dañarse, reemplazando de manera natural y permanente el órgano perdido.

El objeto de este artículo es el describir una ruta segura de acuerdo a los protocolos de los Drs. Radford Tanzer y Burt Brent, quienes gracias a sus capacidades artísticas y quirúrgicas, son hoy el paradigma de la reconstrucción auricular en los casos de pérdida, sin importar su etiología.

\section{Material y Método}

Para el presente artículo analizaremos el desarrollo de casos clínicos que espero ilustren de manera clara la conducta a seguir en cada instancia, como son; el paciente que presenta una microsomía hemifacial, la amputación traumática de la oreja y el manejo de la reconstrucción auricular cuando la piel de la región mastoidea ha sufrido un daño severo que impide usarla para la reconstrucción.

\section{La primera consulta}

Cuando estamos frente a un paciente que padece la ausencia de una oreja, debemos tener en cuenta que sufre las consecuencias de una importante anomalía: si es un niño en edad escolar, la sensación de ser diferente de los demás, algo como incompleto y si ya es mayorcito, el haber tenido que tolerar las bromas y comentarios de sus compañeros de estudio. En el caso de los adolescentes, algunos habrán aprendido a sobrellevar su defecto, pero en la mayoría se puede apreciar una timidez aumentada por la sobreprotección paterna en unos casos y en otros por una clara actitud desafiante o agresiva de un muchacho que no tolera chistes o apodos sobre su defecto. En los casos de trauma, encontramos un paciente generalmente ansioso y molesto por la desfiguración permanente causada por la pérdida de su apéndice auricular y la profunda duda de si este defecto podrá ser corregido o se tendrá que resignar a su deformidad de por vida.

Es importante mostrarse comprensivo con estos pacientes y explicarles los principios generales del procedimiento, las secuelas, cómo son las cicatrices y las posibles deformidades asociadas de la parrilla costal, los verdaderos alcances de la cirugía, las incomodidades del postoperatorio (dolor torácico en las 2 ó 3 primeras semanas del postoperatorio del primer tiempo quirúrgico) y por ultimo, mostrar resultados de casos similares para que el paciente o sus padres se puedan hacer una idea de lo que realmente pueden esperar de la cirugía. Al final se les deben dejar muy claras las posibles complicaciones del procedimiento y hacer énfasis en las realidades de los injertos, como son la posibilidad de reabsorción al no integrase si sufren infección, la muy posible presencia de hematomas que obliguen a su drenaje en una segunda cirugía inmediata y que serán al menos necesarios 4 tiempos quirúrgicos con un intervalo no menor a 3 meses entre uno y otro. En nuestra practica, acostumbramos en este momento a 


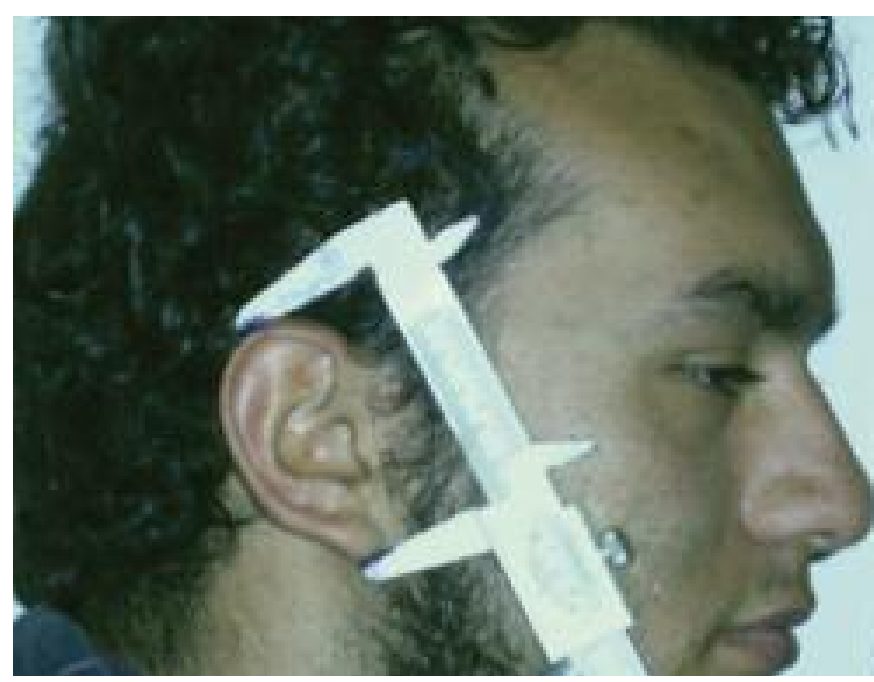

Fig. 1 a. Tamaño y orientación del eje mayor de la oreja.

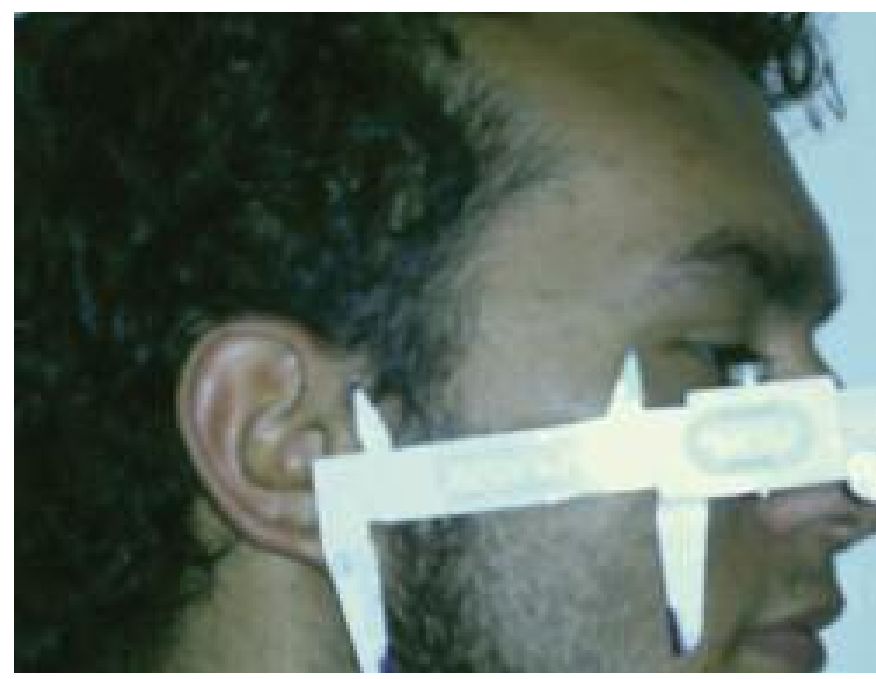

Fig. 1 b. Ubicación del pabellón auricular con respecto al canto externo del ojo.

hacer un estudio fotográfico del paciente y de su defecto y a solicitar las pruebas prequirúrgicas de rutina además de un uroanalisis y una ecografía renal debido a la muy frecuente asociación entre malformaciones auriculares y renales. El paciente es visto en una segunda consulta en la que se revisan los resultados de estos exámenes y si están dentro de límites normales, preguntaremos y aclararemos de nuevo las dudas respecto al procedimiento. Luego se programa la fecha de la cirugía y se planifica la misma; se preparan los modelos y se toman las medidas a cada paciente, que quedan registradas en la historia clínica para el día de la cirugía.

\section{Planificación de la Reconstrucción Auricular}

\section{MICROTIA}

Para ilustrar el modelo general de planificación de la reconstrucción auricular, nos basaremos en un caso de microtia, pues en general, reúne los tipos más complicados en reconstrucción debido a que para lograr el nuevo pabellón auricular se deben seguir y hacer al menos 4 intervenciones diferentes. La microtia se considera, después de la fisura labio-palatina, la segunda malformación congénita más frecuente con una incidencia de 1: 4000 nacimidos vivos; puede presentarse sola o en combinación con otros síndromes (Treacher- Collins, Goldenhar entre otros). Puede ser unilateral, con predominio en varones de $2: 1$ y la relación de aparición derecha - izquierda y bilateral es $5: 3: 1$.

En todos los casos se aprecia una asimetría facial, de mayor o menor grado según la severidad de la malformación, bien aparente en al menos el 50\% de los pacientes. También puede ir acompañada por hemiparesia del VII par del mismo lado de la lesión (2). En el examen clínico se debe tener en cuenta la longitud de la oreja sana; en general las dimensiones de la oreja se hallan dentro de un rango entre 5.5 y $7 \mathrm{~cm}$. de largo por $3.5 \mathrm{~cm}$. de ancho. El lóbulo auricular generalmente se localiza 4 ó $5 \mathrm{~mm}$. por debajo de la implantación del lóbulo de la oreja sana. La implantación superior del hélix, en términos generales, se localiza a unos $6 \mathrm{~cm}$. del canto externo del ojo y la inclinación sigue de una manera general la misma del dorso nasal (Fig.1).

\section{¿Cuándo practicar la reconstrucción auricular?}

Si bien es cierto que técnicamente se podría construir un marco auricular en niños menores de 4 años, desde el punto de vista práctico y real la reconstrucción se puede realizar de manera mas adecuada cuando el niño ha alcanzado una talla de $1.15 \mathrm{~cm}$. de estatura, un peso de entre 25 y $30 \mathrm{Kg}$. o unos $60 \mathrm{~cm}$. de perímetro torácico, medidas que se encuentran en niños eutróficos desde los 6 a 7 años de edad. Lo ideal es iniciar la cirugía entre los 9 y 10 años, debido a que en esta edad ya podemos estar más seguros de encontrar unos cartílagos costales de tamaño adecuado para una adecuada reconstrucción auricular, además de poder gozar de una mejor colaboración del paciente, más consciente a esta edad de su problema.

El planteamiento quirúrgico se inicia calcando las plantillas de la oreja sana en láminas de radiografía limpias y se recortan los moldes que servirán para tallar la forma de la oreja sobre los bloques de cartílago tomados de la parrilla costal (Fig.2). Para poder tener un modelo tridimensional del pabellón auricular a modo de referencia en el momento de la elaboración del molde de cartílago, tomamos impresiones de la oreja sana y luego sacamos un positivo en acrílico dental que podemos consultar siempre que sea necesario (Fig.3). Estos modelos en acrílico tienen la ventaja de poder esterilizarse a gas o en líquido para ser manipulados durante la cirugía. 


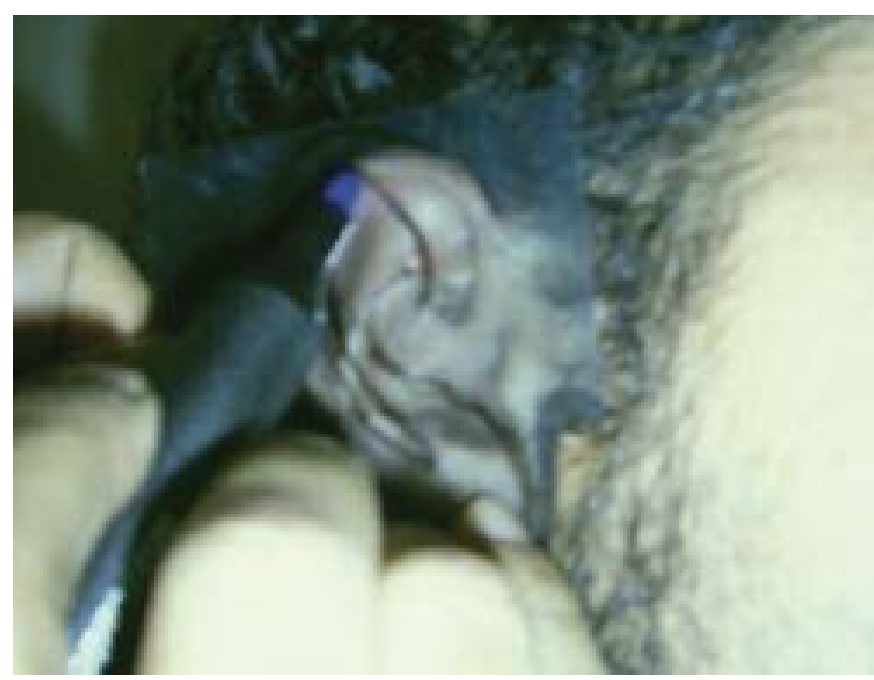

Fig. 2. Calco de la forma de la oreja sobre una lámina de radiografía limpia.

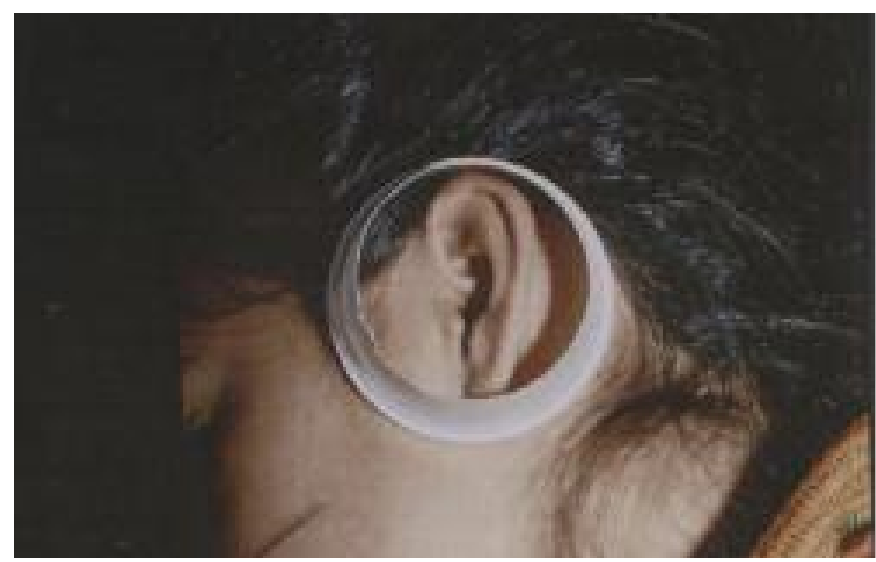

Fig. 3 a. Uso de un vaso desechable como cubeta de impresión en la oreja sana.

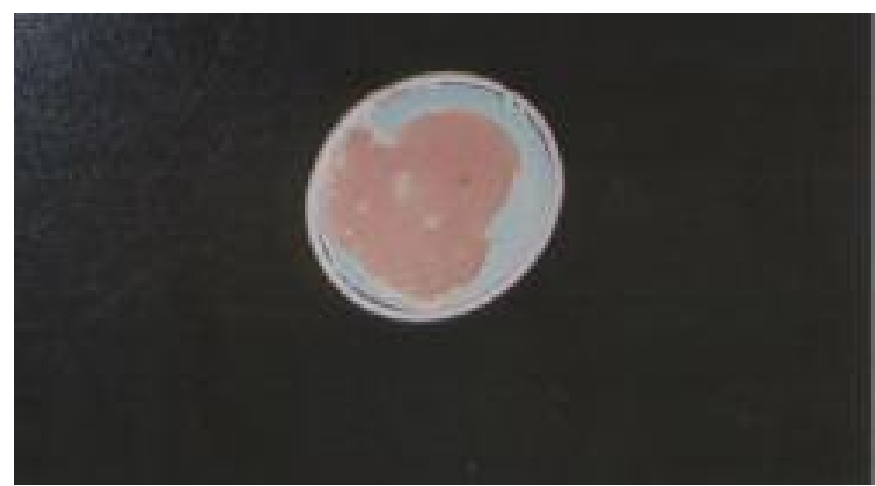

Fig. 3 b. aspecto de la impresión auricular tras el vaciado en acrílico.

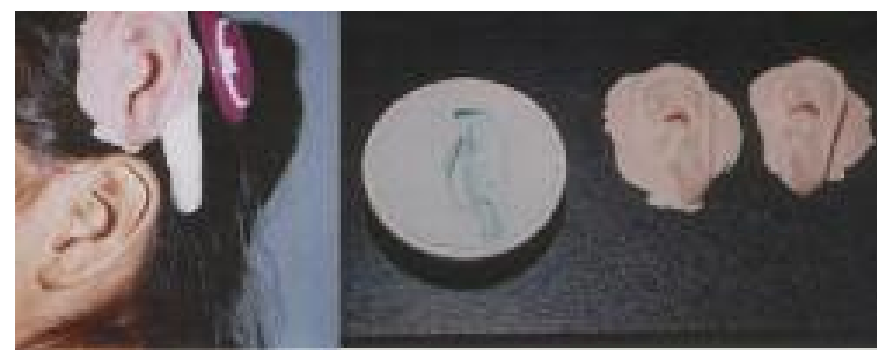

Fig. 3 c. Resultado del molde. Se pueden sacar varias copias si es necesario.
Técnica quirúrgica del primer tiempo de la reconstrucción auricular

Bajo anestesia general, se practica asepsia de ambos campos operatorios. Empleamos el tórax contralateral a la lesión, pues como está descrito por Brent la natural curvatura de los arcos costales va a contribuir al tallado y conformación del molde del pabellón de la oreja (esto claro está, no excluye el poderlo tallar del mismo lado si por alguna razón se tuviera que hacer). Se inicia el tratamiento antibiótico con Cefazolina $50 \mathrm{mg} / \mathrm{Kg}$. en niños o $1 \mathrm{gr}$. IV en adultos durante la inducción anestésica; debido al tiempo prolongado de la cirugía y en consideración al material de injerto, estos antibióticos no los consideramos como profilácticos, sino terapéuticos y se continuarán usando 5 días más después de la cirugía. También se practica la limpieza del lado afectado de la microtia, aunque debido al tiempo inicial de toma de los injertos de cartílago, la talla del molde y su implantación, es conveniente volver a lavar el sitio del defecto cuando se vaya a trabajar sobre esa zona. Antes de poner los campos, infiltramos la pared torácica con 10 cc. de solución al $0.5 \%$ de lidocaina con epinefrina al 1: 200.000 a lo largo del sitio planeado para la incisión a fin de disminuir el sangrado.

La incisión se inicia cerca de la unión esternal, a nivel del $6^{\circ}$ espacio intercostal y ligeramente diagonal, siguiendo el reborde costal hasta el punto donde se inicia la curvatura normal de la $8^{\mathrm{a}}$ costilla (Fig.4). Nos permite acceder fácilmente a los tres arcos costales, a saber, la sincondrosis de la $6^{\mathrm{a}}$ y $7^{\mathrm{a}}$ costillas y la $8^{a}$ costilla. La disección de los cartílagos costales, se inicia tomando primero la $8^{\mathrm{a}}$ costilla, maniobra que facilita luego la disección de los cartílagos costales a nivel de la sincondrosis. En lo posible, esta disección debe hacerse con mucho cuidado, preservando el pericondrio y sin lesionar la pleura, pero si se llegara a lesionar, debemos localizar la perforación, introducir una sonda de Nélaton calibre 12 en la herida y

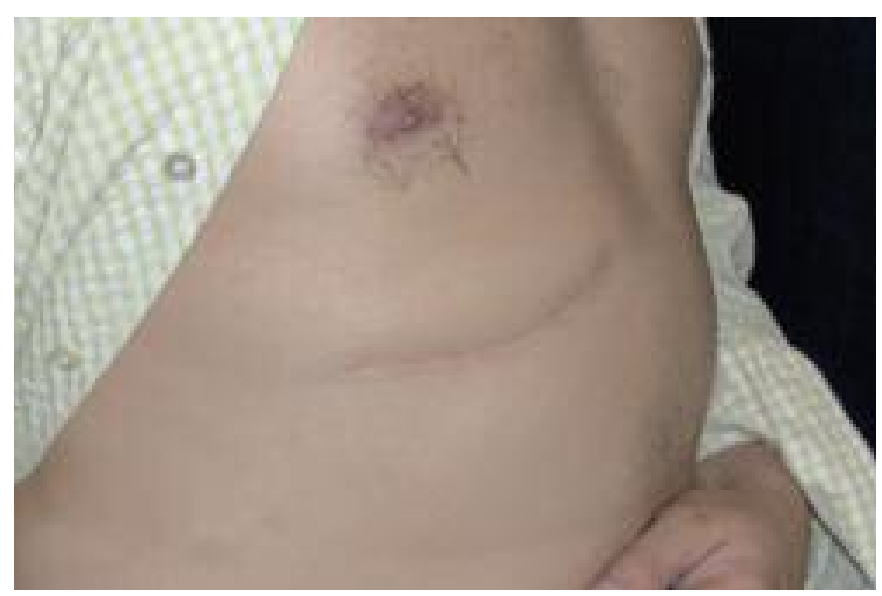

Fig. 4. Localización de la incisión para la toma de injertos costales y cicatriz resultante. 


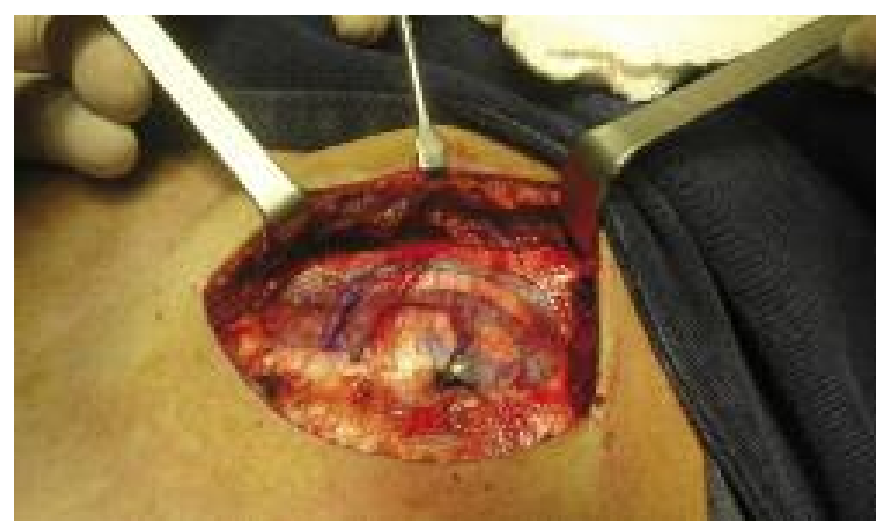

Fig. 5. Zona donante y uso de los moldes para determinar la cantidad de cartílagos costal necesario.

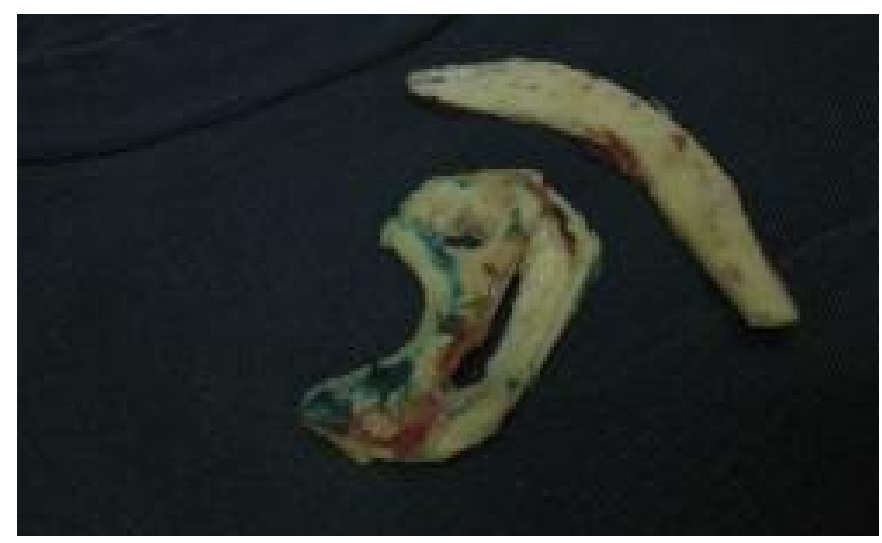

Fig. 6. Diferentes piezas del cartílago costal talladas antes de su ensamblaje.

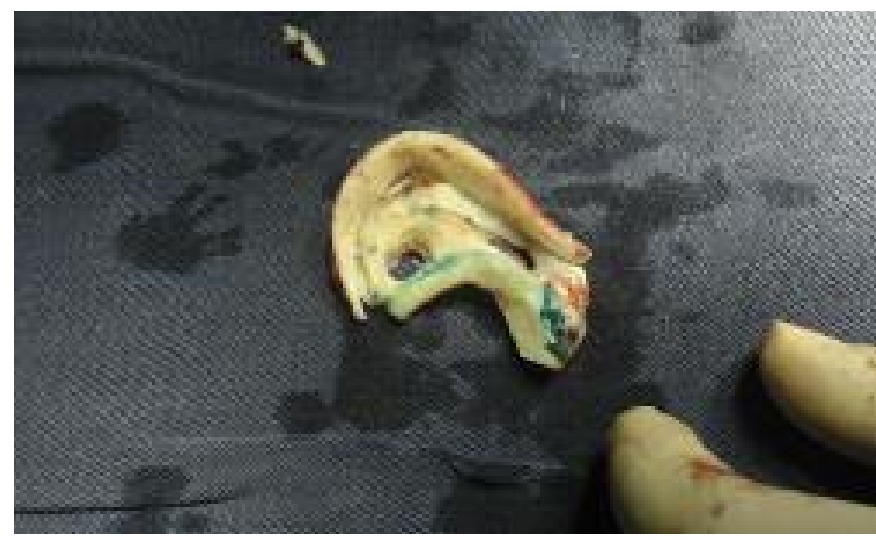

Fig. 7. Piezas ensambladas.

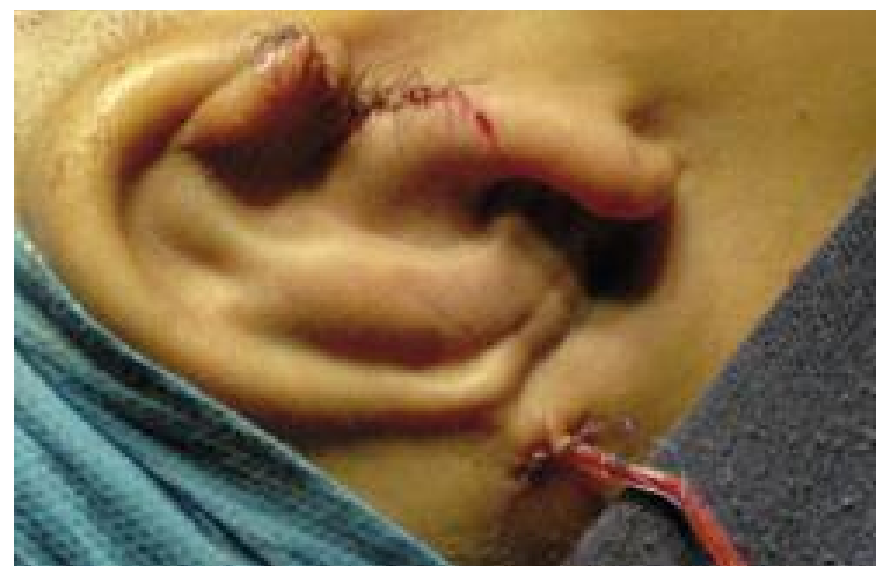

Fig. 8 a. Cateter para drenaje al vacío y su fijación en la piel. suturar alrededor de manera que quede hermética; luego, sumergido el extremo libre de la sonda en solución salina (trampa de agua), solicitar la evacuación del aire de la cavidad pleural por parte del anestesista y luego extraer la sonda y proceder al cierre por planos de la pared torácica. Es conveniente tomar una radiografía de tórax en quirófano para constatar la adecuada expansión pulmonar.

Una vez tomados los injertos, tallamos en una mesa auxiliar los moldes de cartílago y entre tanto el ayudante procederá al cierre por planos de la herida torácica, ahorrando tiempo operatorio.

Como complemento al cierre, es adecuado practicar una infiltración de los bordes de la herida con solución de bupivacaína con epinefrina para mitigar el dolor postoperatorio.

\section{Tallado del molde de cartílago auricular.}

Limpiamos de tejido muscular los especímenes de cartílago dejando solo el pericondrio. Luego, pintamos sobre la superficie de la sicondrosis de la $6^{a}$ y $7^{a}$ costillas el diseño de lo que será la base del molde y los relieves del antihélix (Fig. 5); esta talla se puede hacer por medio del uso combinado de hojas de bisturí $\mathrm{n}^{\circ} 11,15$, y 23 o si se tienen a mano como describe Brent, con escoplos acanalados; lleva algún tiempo adquirir la destreza necesaria para tomar, tallar y ensamblar un molde adecuado, por lo que se recomienda practicar en cadáveres antes de pasar a la práctica clínica. Una vez tallado el injerto que será la base, se talla el cartílago de la $8^{\text {a }}$ costilla, adelgazándolo hasta lograr una tira de cartílago lo suficientemente flexible para arquearla alrededor del molde de la base y fabricar el helix auricular, tratando de que sea lo más prominente posible (Fig.6). Cuando se han tallado ambas piezas, se ensamblan por medio de suturas de nylon 4/0, dejando los nudos en la cara profunda del molde para que no se transparenten o se expongan en la piel (Fig. 7). Como el lograr la confección de un buen marco auricular comprende al menos el $60 \%$ de la ecuación: injerto + piel $=$ oreja, se recomienda usar todo el tiempo que se crea necesario para lograrlo. Una vez formado el marco básico de la nueva oreja, escogemos de entre los remanentes una pieza adecuada para agregar en forma de cuña a la cara profunda del molde, a fin de dar proyección a la concha auricular (2-4).

El marco auricular ensamblado se coloca en solución salina para evitar la desecación del injerto y se procede con la segunda parte de la ecuación: la conformación de un adecuado bolsillo de piel en la región mastoidea, con capacidad suficiente para recibir cómodamente el injerto de cartílago y nutrirlo para su adecuada integración. 


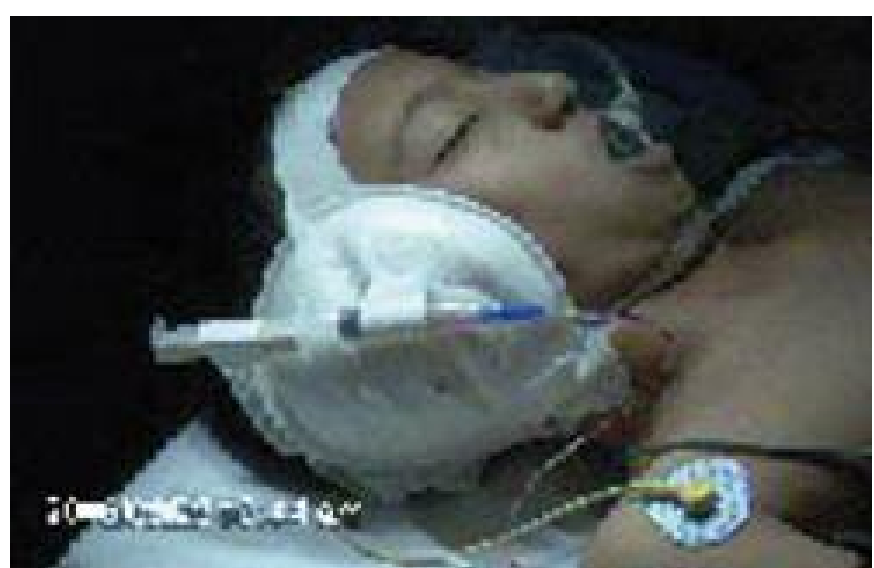

Fig. 8 b. Sistema de evacuación al vacío montado en una jeringa de $10 \mathrm{cc}$.

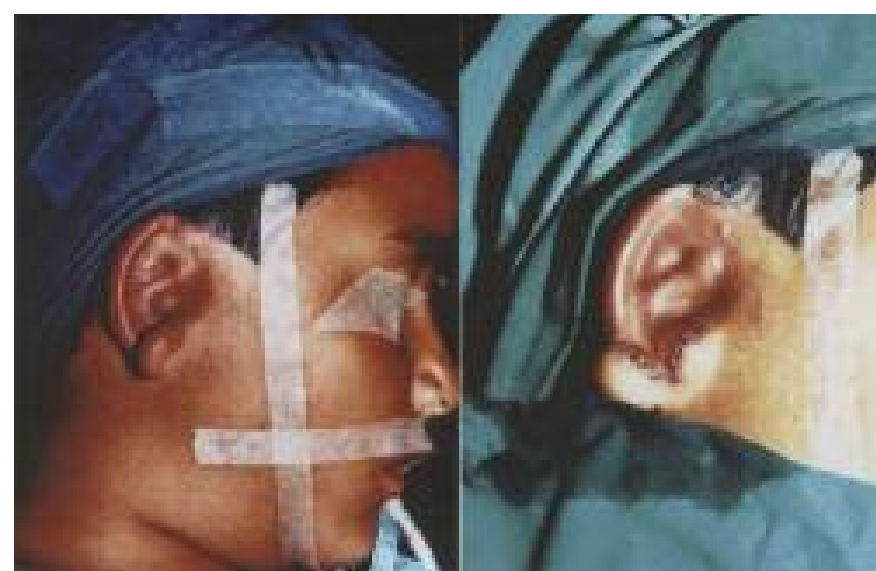

Fig. 9 a. Pre y postoperatorio inmediato de la trasposición del lóbulo auricular.

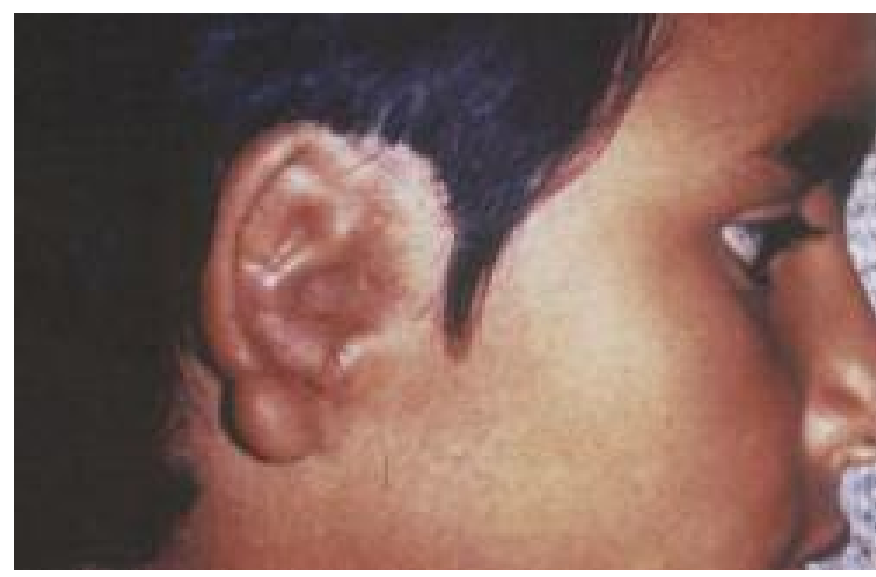

Fig. 9 b. Aspecto de la oreja a los 6 meses con el lóbulo traspuesto una vez cicatrizado.

\section{Conformación del bolsillo de piel}

Preparamos nuevamente el campo operatorio del lado del defecto. Se dibuja una incisión en el borde anterior de los remanentes de cartílago auricular y sin utilizar ningún tipo de vasoconstrictor, se procede a disecar los restos malformados de cartílago en un plano superficial, pero sin dañar la piel o su irrigación en el plano subdérmico. Continuamos por este mismo plano hasta conformar un bolsillo de piel en el área marcada previamente de acuerdo a nuestro diseño del sitio de la nueva oreja.

La disección del bolsillo se extiende al menos $1 \mathrm{~cm}$. más allá de lo marcado o lo que sea necesario para recibir el molde sin presiones. Con la ayuda de una luz frontal practicamos una muy minuciosa hemostasia para evitar una de las complicaciones más comunes, el hematoma. Luego, trataremos de implantar el molde introduciéndolo con cuidado por su extremo más delgado y girándolo hacia el área que correspondería al lóbulo; si se desliza suavemente y se puede introducir fácilmente, el tamaño y forma del bolsillo son los adecuados; revisamos la localización tomando como referencia el trazo previo y los puntos de referencia anatómicos. Si el molde no entra fácilmente, lo retiraremos, revisaremos el bolsillo en busca de bridas o adherencias residuales o si es necesario ampliaremos la disección del bolsillo y volveremos a revisar la hemostasia.

Una vez superado el proceso, aplicamos un sistema de drenaje al vacío (Fig. 8 a), siguiendo la técnica de Brent, tomamos un equipo de punción venosa de mariposa cuya modificación consiste en que de su extremo se retira la aguja y se practican varios orificios en la manguerita de plástico. Por una incisión por contrabertura se introduce este extremo del catéter y se sitúa por debajo del molde de cartílago. El extremo con el acople se fija a una jeringa de $10 \mathrm{cc}$ y se retiene el émbolo por medio de un depresor lingual. Esta variación es más practica que la de utilizar los tubos de vacío de Brent. Una alternativa sería también una sonda de Nélaton $\mathrm{n}^{\circ} 8$. A veces queda la tentación de aumentar el vacío con una jeringa de $20 \mathrm{cc}$ o de $50 \mathrm{cc}$ para que sea más efectivo; el resultado sería que el exceso de vacío podría producir una necrosis de piel a nivel de los rebordes más prominentes del molde y crear sufrimiento de la piel y esfacelación a este nivel. Tras algunos casos y de manera empírica, descubrimos que la jeringa de $10 \mathrm{cc}$ produce el suficiente vacío sin comprometer la circulación de la piel.

Se procede entonces al cierre de la herida sin tensión con sutura de monofilamento nylon o polipropileno 5/0 o 6/0 con aguja cortante. Con frecuencia, la piel no es suficiente para lograr un cierre sin tensión; entonces se aplica un injerto de piel de espesor total de la región retroauricular del lado sano y se fija con un vendaje de capitoneo. Activamos el vacío que va a dibujar el relieve del molde bajo la piel y aplicamos gasas impregnadas en antibiótico para copiar los relieves y sobre ellas, gasas extendidas para formar un vendaje oclusivo de presión muy moderada cubiertas por un protector de oído, dejando por fuera y en posición estratégica nuestro sistema de vacío a jeringa (Fig. 8 b). Es esencial instruir al personal sanitario en 
el manejo del sistema de vacío y las precauciones que han de tener con el mismo.

A las 24 horas se practica la primera cura para revisar que no haya hematomas y que la circulación del colgajo de piel sea adecuada. Se vuelve a poner el vendaje otras 72 horas al cabo de las cuales se cita al paciente en el consultorio y se retiran el vendaje y el sistema de vacío. En este momento, generalmente la oreja nueva está edematosa y en ocasiones no se aprecia mucho la forma a pesar de haberse mantenido adecuadamente el vacío, pero irá recuperando su forma a medida que pase el edema. Se debe vigilar eso sí que el edema no se acompañe de eritema severo, flogosis o fiebre, pues estaríamos ante una infección que se debe tratar de manera agresiva, incluso se debe pensar en lavados quirúrgicos ínterdiarios de la cavidad hasta controlarla y salvar la reconstrucción. El régimen de antibióticos se deberá instaurar de acuerdo a los resultados del cultivo y antibiograma.

Tras sanar las heridas quirúrgicas sin complicaciones, el paciente puede reintegrarse a sus actividades normales excepto deportes de contacto, que debe evitar los 2 primeros meses así como tener cuidado de golpes o traumatismos severos.

\section{Segundo tiempo de la reconstrucción auricular: rotación del lóbulo auricular, tres meses más tarde}

En esta segunda etapa nos enfrentamos a poner el complemento ideal al marco que ya hemos construido; se trata de practicar una Z-Plastia en la cual uno de los colgajos es el remanente del lóbulo de la oreja de la deformidad inicial. Esta cirugía, en personas mayores, se puede llevara acabo bajo anestesia local y sedación y en los niños bajo anestesia general.

Lo primero será el diseño de la nueva disposición del lóbulo con referencia al marco implantado. Dibujamos el tejido lobular disponible y tallamos este brazo de la zplastia. A continuación, rotamos sobre el polo inferior del pabellón auricular y nos fijamos en cuál es la posición mas armónica del lóbulo; entonces diseñamos la incisión que va a conformar el otro brazo de la z-plastia y llevamos a cabo la transposición de los tejidos. Para la implantación del lóbulo, en su unión con el reborde del hélix, practicamos un cierre en w-plastia con el fin de evitar una banda de constricción de la cicatriz que se formaba en nuestros primeros casos y provocaba una cicatriz muy aparente. El cierre se hace con sutura de monofilamento $6 / 0$ y dejamos un vendaje de gasa durante 2 días; los puntos se retiran a los 5 ó 6 días (Fig. 9) $(3,4)$.

\section{Seis meses después, la recta final: elevación del pabellón de la oreja, tercer tiempo operatorio}

Si con los dos tiempos anteriores hemos logrado una buena proyección y definición de la oreja, este

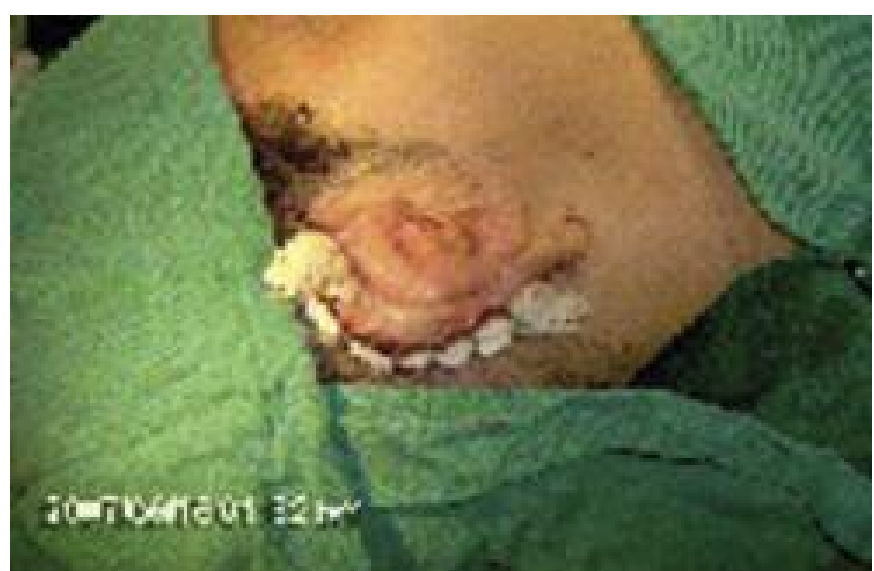

Fig.10 a. Imagen del pabellón auricular tras ser elevado y situación del injerto de piel total.

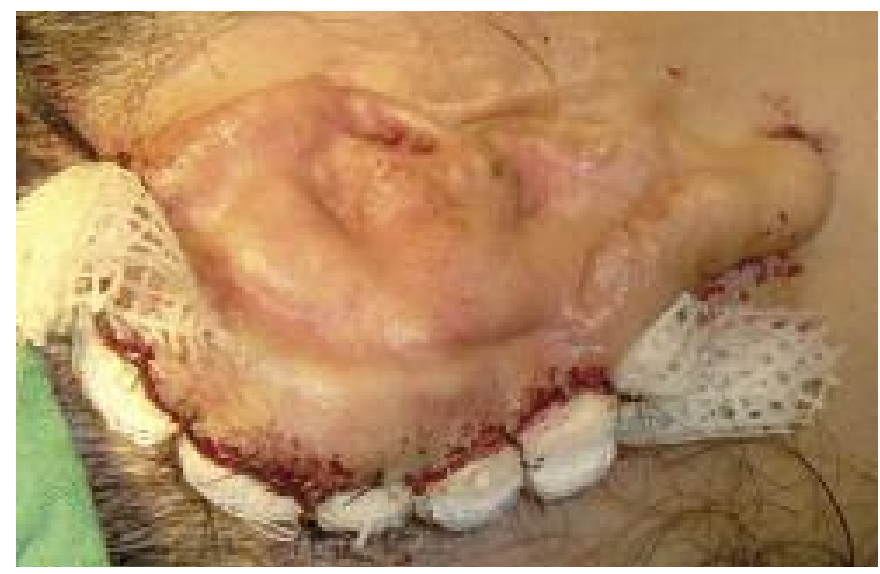

Fig. 10 b. Vendaje de capitoneo al final de la cirugía, tras reconstrucción del surco retroauricular.

tercer tiempo puede obviarse. Si por el contrario la oreja quedó con poca proyección se puede destacar su presencia creando un surco retroauricular y haciéndola más aparente y mejor definida, con lo que se aumenta su proyección en el cráneo. Esta cirugía se lleva a cabo bajo anestesia general.

Dibujamos una incisión sobre la piel mastoidea, 5 $\mathrm{mm}$ por fuera del relieve del pabellón auricular. Infiltramos la piel en la línea de incisión con una solución de lidocaina al $0.5 \%$ con epinefrina al 1:400.000, así como por debajo del injerto de cartílago tratando de producir una disección hidráulica del plano; incidimos la piel hasta el plano por encima de la fascia mastoidea, por encima del cual se inicia el despegamiento del pabellón auricular, teniendo especial cuidado de identificar la cápsula de tejido fibroso que se ha formado alrededor del cartílago para protegerla y preservar indemne este tejido que va a recibir el injerto de piel necesario para la cara posterior del pabellón auricular una vez levantado. Si se llegara a herir esta cápsula, sería necesario repararla muy cuidadosamente. El pabellón auricular se levanta entonces hasta un punto en el que se identifica la nueva concha auricular y todo el vértice del helix y el lóbulo. Se practica 


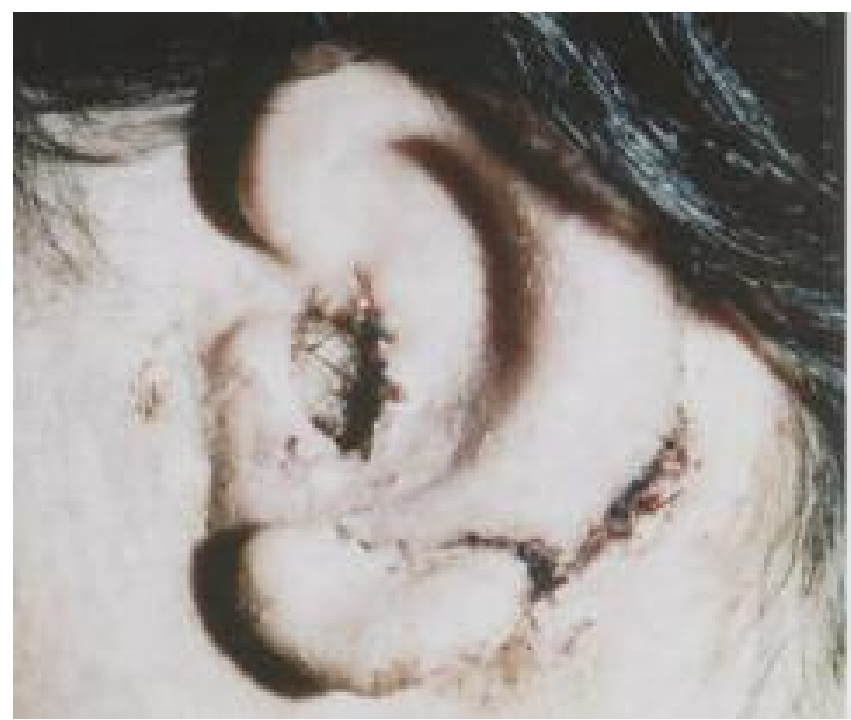

Fig. 11. Aspecto de la oreja y del trago reconstruido según método de Kirkham.

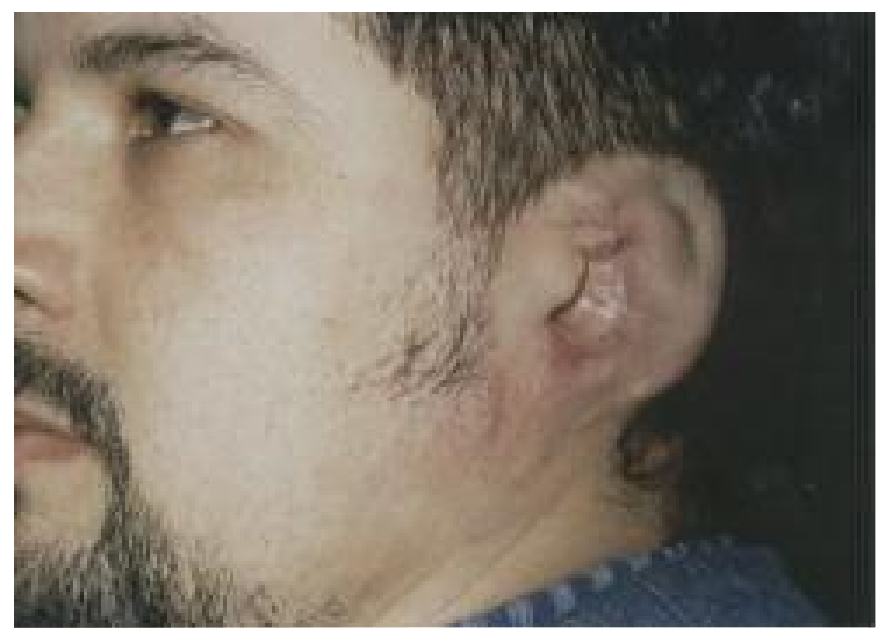

Fig. 12 a. El pabellón auricular después del primer tiempo quirúrgico ( 6 meses postoperatorio) y con cabellos en el hélix.

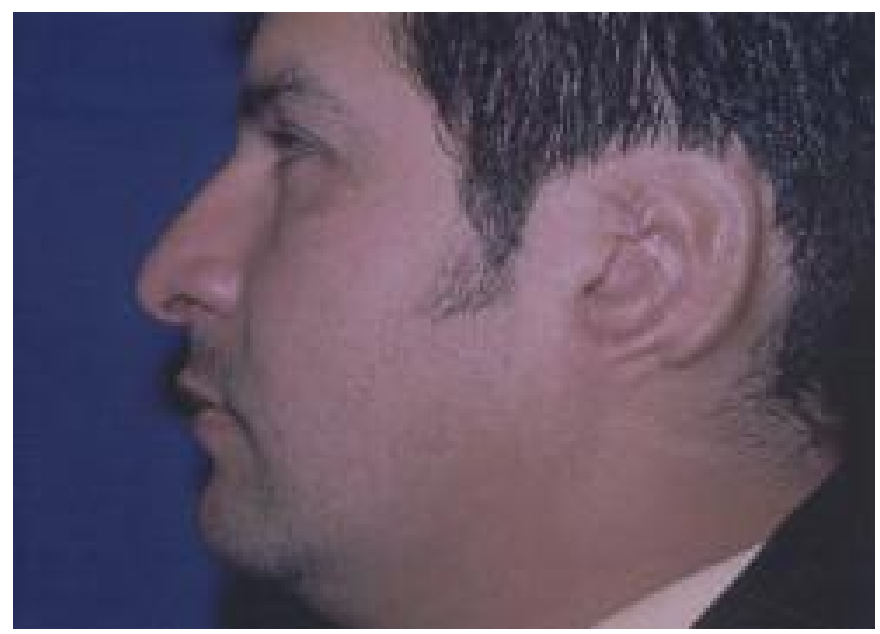

Fig. 12 b. El mismo paciente después de depilación láser de la oreja y del reborde del pelo.

hemostasia minuciosa y se procede a liberar un poco la piel posterior a nivel mastoideo, creando un colgajo por deslizamiento y avanzamos lo más que se pueda esta piel mastoidea hacia el defecto creado al levantar el pabellón de la oreja. Este colgajo se avan- za y se fija con puntos de Vycril 4/0 en U de la dermis a la fascia mastoidea. Una vez practicada esta maniobra, se procede a medir el defecto resultante y se toma la medida para el injerto de piel de espesor total, que de acuerdo al tamaño, podrá tomarse ya sea de piel retroauricular de la oreja contralateral, a nivel del pliegue inguinal o de la cara interna del brazo en su tercio superior. Si bien la región supraclavicular es una de las zonas clásicas de toma de injertos de piel total fina, debido a su situación expuesta no es un lugar de elección en estos casos. Luego el injerto se fija a los bordes del defecto con vendaje de capitoneo que se dejará durante 8 días (Fig. 10).

\section{Construcción del trago}

Existen dos medios eficaces para reconstruir el trago. El primero es el método de Kirkham que consiste en practicar un colgajo en $U$ de base anterior y plegarlo sobre sí mismo. Se puede profundizar la concha auricular resecando el tejido fibroso del área cruenta creada y aplicando un injerto de piel de espesor total y se puede incluir entre las dos hojas del colgajo un pequeño injerto de cartílago de forma y tamaño adecuados para que ayude a definir la forma del trago (Fig.11) $(3,5)$. El segundo, ideado por Brent, es el practicar a nivel del sitio del trago una incisión en J y disecar la piel anterior un poco para a continuación tomar una injerto compuesto (piel y cartílago) de forma elíptica de la cara anterior de la concha sana que se sutura en la herida creada, conformando de esta manera un trago (2.3) Ambos métodos son satisfactorios.

Una vez finalizados estos 4 tiempos quirúrgicos, podemos decir que ya tenemos una oreja. Sin embargo la mayoría de las veces quedan detalles; el más notorio es la inclusión de piel cabelluda en el tercio superior del pabellón auricular. El método de elección para solventar este problema es en la actualidad la depilación definitiva con láser, que puede llevarse a cabo en cualquier momento de la reconstrucción si bien hay dos momentos ideales para hacerla. El primero cuando se está planeando la cirugía y antes del primer tiempo quirúrgico, en que se diseña el área de piel lampiña y el recorte de la patilla, así una vez se inicia el proceso de reconstrucción se cuenta con una amplia zona de piel sin cabellos (6). El otro, cuando ya se ha hecho el primer tiempo y se puede delimitar el área pilosa de la oreja, entonces con la oreja en su sitio, se puede practicar la depilación definitiva y conformar una línea del pelo y de la patilla sobre medidas (Fig. 12).

Cuando se siguen los tiempos quirúrgicos y no se presentan complicaciones se obtienen muy buenos resultados, logrando reconstruir por completo un 

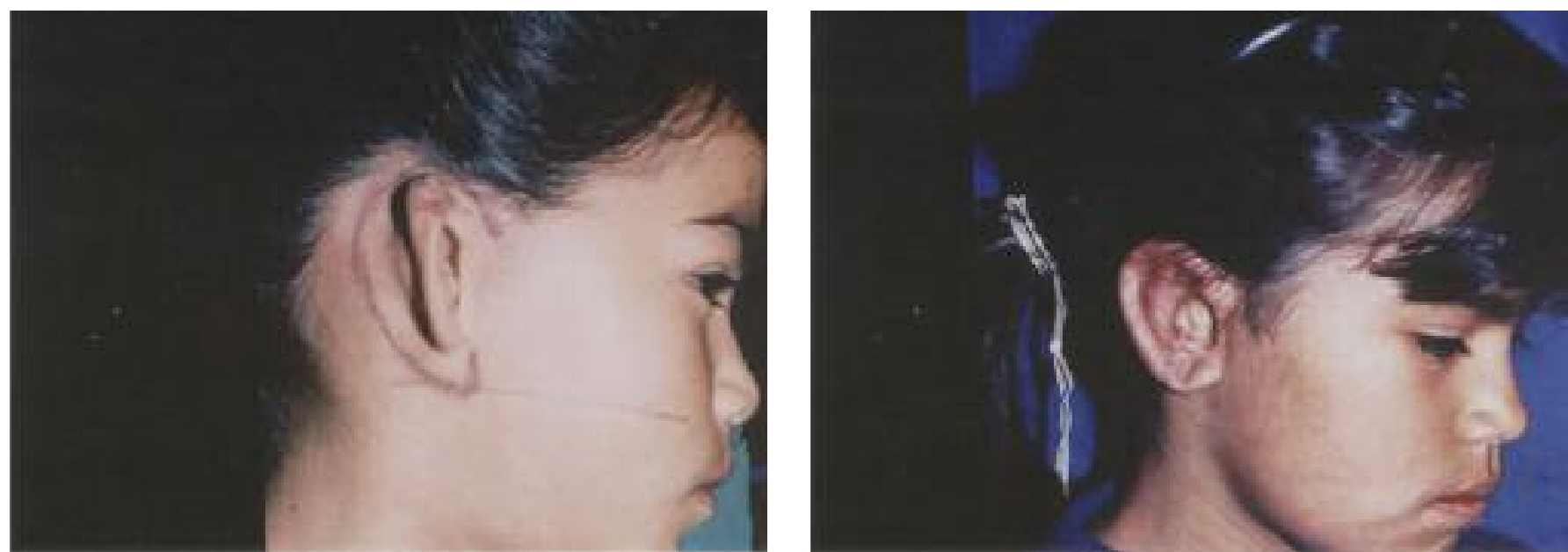

Fig. 13. Pre y postoperatorio a los 18 meses de una paciente con reconstrucción auricular según técnica de Brent.

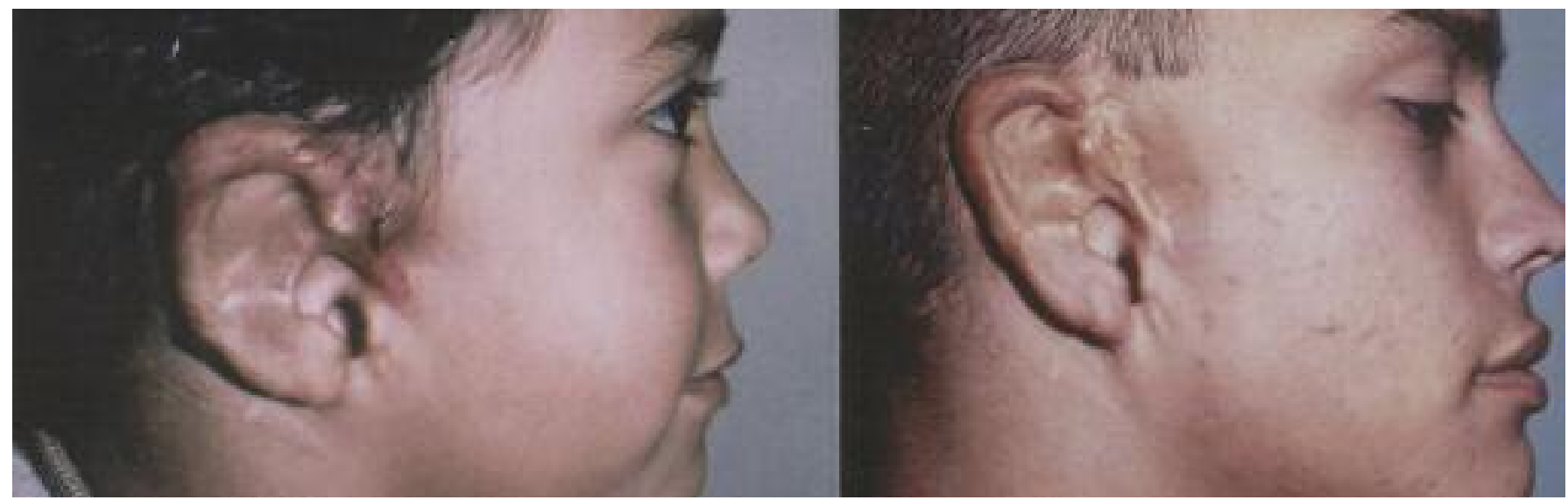

Fig.14. Paciente con reconstrucción auricular a los 7 años y 12 años después.

pabellón auricular bien situado, con aspecto natural y capaz de tolerar las condiciones diarias y el paso de los años (Fig. 13, 14).

\section{RECONSTRUCCIÓN DE LA OREJA AMPUTADA}

Cuando se produce la amputación de una oreja, ya sea de forma traumática o quirúrgica por cáncer pero queda la piel adyacente del área mastoidea en buenas condiciones, se puede proceder a una reconstrucción auricular como si fuera una microtia. En caso de no tener el lóbulo, se debe tener en cuenta que hay que

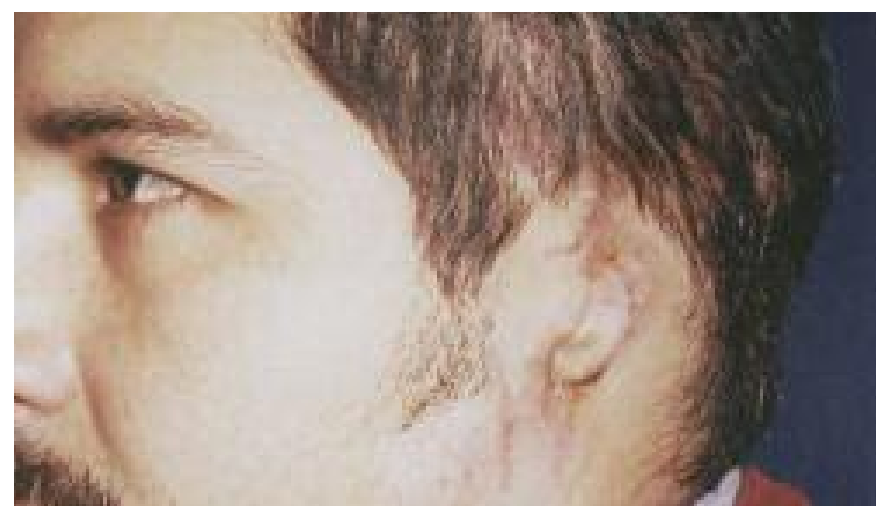

Fig. 15 a. Paciente con amputación completa de la oreja incluido el lóbulo. tallar en el marco de cartílago el lóbulo auricular para que cuando se eleve el pabellón auricular se eleve también la parte del lóbulo y no tener que reconstruirlo en otro tiempo quirúrgico. Como en estos casos no hay pérdida del trago y el conducto auditivo externo ya está, la reconstrucción se limita a dos tiempos quirúrgicos (Fig. 15). En algunas ocasiones existe el lóbulo, que puede rotarse en el primer tiempo quirúrgico en el momento de la implantación del marco de cartílago.

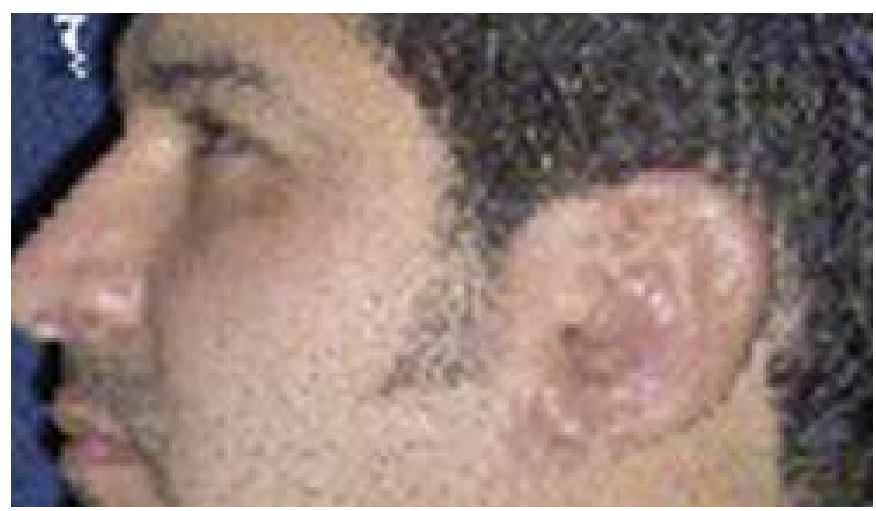

Fig. 15 b. el mismo paciente al año de postoperatorio. 


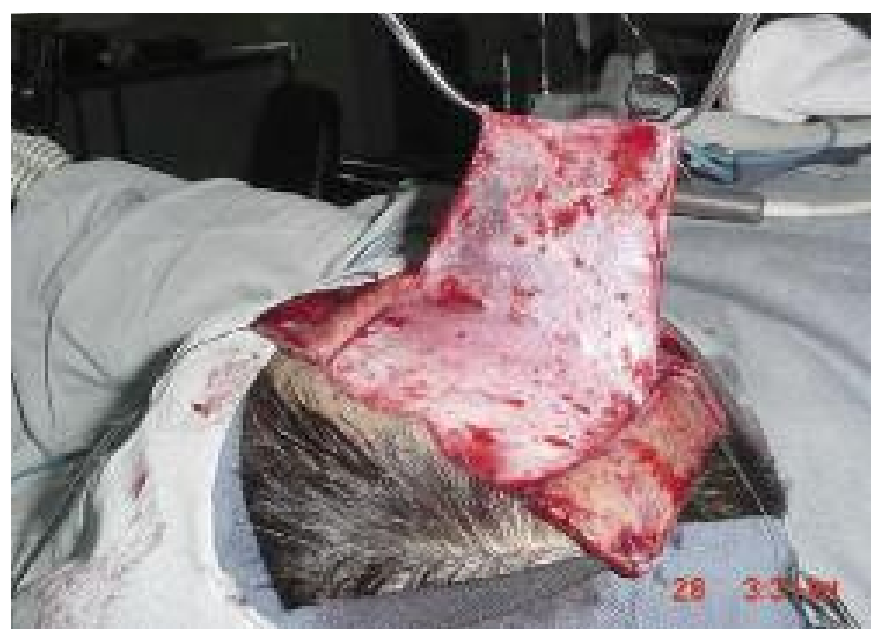

Fig. 16. Disección del colgajo de fascia temporal.

\section{Reconstrucción secundaria de la oreja: qué hacer cuando la primera vez no resultó bien}

Cuando se obtiene un mal resultado en la reconstrucción auricular, ya se ha dañado el colgajo de piel mastoidea y / o se ha reabsorbido el cartílago dejando una piel con fibrosis cicatricial y adherente, o cuando por un accidente se dañó también la piel mastoidea ¿qué hacer?

Lo primero será valorar en el área preauricular el pulso de la arteria temporal superficial para continuarlo hasta el área temporal, al tiempo que revisamos que el cuero cabelludo a este nivel no haya sido también traumatizado. Solicitaremos un doppler vascular de la arteria temporal superficial para verificar su existencia. Podremos iniciar la planificación de la reconstrucción auricular por medio de un colgajo de fascia temporal de Tegtmeier (5); este colgajo se elabora a partir de la muy fina y resistente fascia temporal superficial, que se va convertir en una interfase que por un lado va a permitir la cobertura y alimentación del marco de cartílago auricular, al tiempo que permite la aplicación de una lámina de injerto de piel de espesor parcial que proveerá la cubierta cutánea.

La preparación del campo operatorio incluye un corte de cabello corto, incluso en mujeres y su lavado el día antes de la cirugía y en la mañana del día de la operación. En el lado comprometido, se diseña una incisión en $\mathrm{T}$ o en Y y se infiltra la zona con solución al $0.5 \%$ de lidocaina con epinefrina al 1:400.000. Se incide el cuero cabelludo hasta visualizar los folículos pilosos e inmediatamente se aprecia la fascia temporal superficial de color grisáceo; ejerciendo tracción por medio de ganchos de piel, se establece un plano anatómico y con bisturí, se diseca la cara superficial de la fascia de la cara profunda de la piel cabelluda de los labios de la $\mathrm{T}$ hasta una extensión que conforme

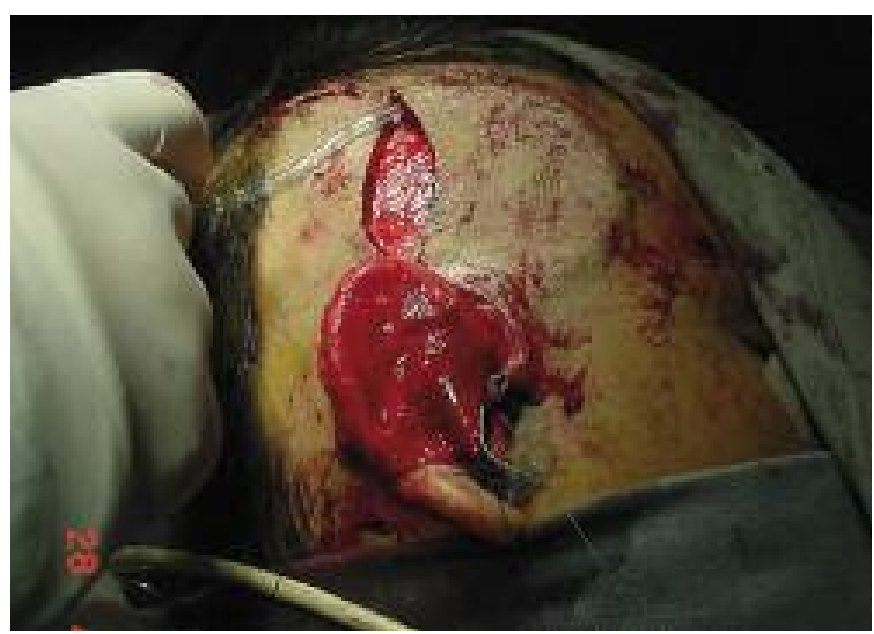

Fig. 17. Colgajo de fascia temporal recubriendo el molde de cartílago.

un colgajo de al menos $5 \mathrm{~cm}$. de ancho por 6 ó $7 \mathrm{~cm}$. de largo, suficiente para cubrir el molde de cartílago que va a conformar el nuevo pabellón auricular (Fig. 16).

Una vez disecada la cara superficial de la fascia, por su borde superior, se inicia la disección y elevación del colgajo de fascia temporal separándola de la fascia temporal profunda del músculo temporal y conformando un colgajo cuadrangular que contenga la arteria temporal superficial, el cual al doblarse sobre su base, cubra el marco de cartílago que se ha colocado sobre el lecho cruento del área auricular de donde se han resecado los restos cicatriciales (Fig.17). Rotamos el lóbulo auricular, si existe y se procede a fijar el colgajo a los remanentes cutáneos forrando el molde de cartílago. Luego se aplican dos sistemas separados de vacío, uno de los cuales es para el lecho donante del colgajo en la región temporal con tubos de un diámetro de $1 / 8$ y una sonda de Nelaton $n^{\circ} 10$ en el sitio de la oreja sujeta a una jeringa de $10 \mathrm{cc}$. A continuación y una vez establecido el vacío, se procede a tomar del la región glútea o superior del muslo una lámina de piel de espesor parcial del ancho necesario para cubrir el colgajo de fascia temporal, que se fijará a los bordes de la herida con suturas reabsorbibles y vendaje de capitoneo y se mantendrá en posición al menos 8 días si no se presentan signos de secreción o mal olor. Pasado ese tiempo se retira el vendaje y se debe apreciar el injerto integrado, tras lo cual se aplicará un nuevo vendaje levemente compresivo. Hacemos nueva cura a los 5 días y ya se puede dejar la nueva oreja descubierta cuidando lubricar la piel del injerto con cremas emolientes. Al cabo de 2 meses el paciente puede realizar todas sus actividades normales; en caso de practicar deportes de contacto se debe instaurar alguna protección de esta oreja (Fig. 18) $(2,5)$. 


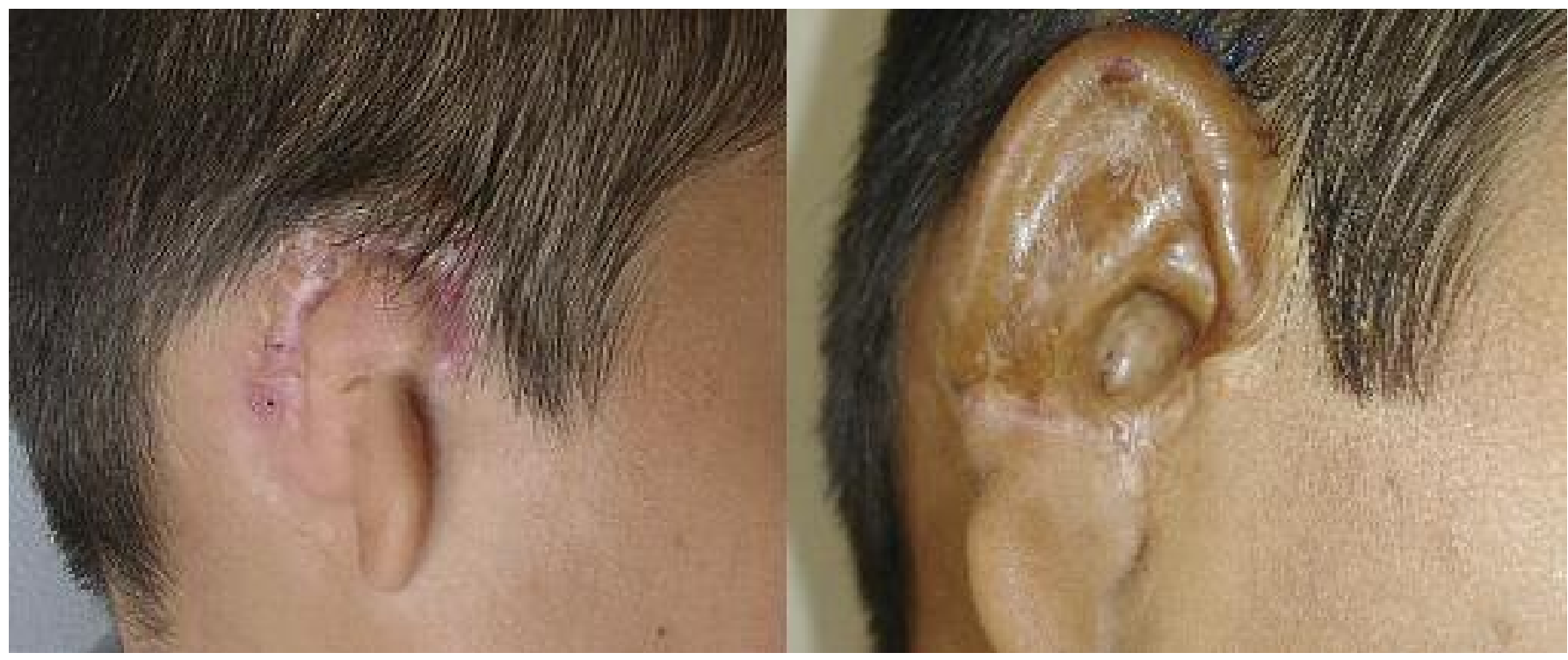

Fig. 18. Pre y postoperatorio a los 2 años de una reconstrucción auricular mediante colgajo de fascia temporal.

\section{Discusión}

Se revisaron 50 historias clínicas correspondientes a los pacientes intervenidos entre los años de 1987 y 2006 en los cuales se había realizado el procedimiento descrito en el Hospital Central de la Policía de Bogotá (Colombia). De estos 50 casos, 12 corresponden al género femenino (24\%) y $38(76 \%)$ al genero masculino. En el rango de edades se constató que aunque algunos fueron vistos por primera vez como lactantes, todos fueron intervenidos después de los 7 años de edad (escolares) 33 casos (66\%), 9 adolescentes entre los 14 y los 18 años (18\%) y 8 adultos (16\%).

En cuanto a la técnica quirúrgica, todos fueron tratados de acuerdo a los protocolos del artículo usando la técnica de Brent, excepto un caso en el que se utilizó un expansor tisular como paso previo a la implantación del injerto de cartílago. En este caso, que evolucionó bien, podemos comentar que realmente no vimos ninguna ventaja para lograr el resultado buscado; más bien se incrementaron los gastos debido al costo del expansor, una intervención de más para la aplicación del mismo, demora de 12 semanas para iniciar el primer tiempo quirúrgico de la reconstrucción auricular, más los riesgos de complicaciones inherentes. En consecuencia, tras este primer caso se suspendió su uso en este tipo de reconstrucciones.

En cuanto a los pacientes que necesitaron el uso de un colgajo de fascia temporal hallamos 4 casos $(8 \%)$; 3 correspondieron a intentos fallidos de reconstrucción auricular llevados a cabo en otras instituciones y 1 caso por amputación traumática de la oreja por explosión de una bomba.

La causa más común de consulta para reconstrucción de la oreja fue la microtia congénita con 43 casos
$(86 \%)$, trauma en 7 casos (14\%) y no hubo casos de reconstrucción debidos a pérdida quirúrgica por cáncer. En cuanto a la presentación, solo se hallaron 2 casos de microtia bilateral en un niño y una niña, curiosamente la niña tenía una hermana mayor con microtia unilateral.

El porcentaje total de complicaciones fue de $16 \%$. Cinco pacientes presentaron sufrimiento de la piel por exceso de presión con necrosis parcial que necesitó tratamiento con colgajos locales en 1 caso y 3 pacientes presentaron infección postoperatoria que evolucionó bien con lavados diarios y antibióticoterapia sistémica. En uno de estos casos se presentó una combinación de necrosis marginal de piel seguida de una infección muy severa que no se controló hasta la eliminación del cartílago, lo cual significó la pérdida del procedimiento. Al final, todos los casos menos uno ya descrito, fueron exitosos a pesar de las complicaciones. La complicación por sufrimiento de piel del colgajo se debió a nuestro juicio a la suma de dos causas: una mala indicación al aplicar un exceso de vacío en la primera cirugía, presión del vendaje o a tratar de poner un molde muy grande dentro de un bolsillo de piel muy estrecho.

\section{Conclusiones}

Tras un detallado análisis de la técnica quirúrgica, probada por 24 años de práctica clínica, creemos que se ha cumplido la premisa fijada por el Dr. Sir Harold Gillies para la reconstrucción auricular: obtener una oreja natural, permanente y bien localizada en la cabeza, capaz de tolerar las actividades diarias de la persona sin que sienta miedo a que se le caiga con los movimientos de la cabeza. El procedimiento no es 
sencillo, pues consta de 4 fases en todas de las cuales se debe tener sumo cuidado y siempre teniendo en cuenta la ecuación de piel + cartílago $=$ oreja.

Dentro de las variables, debemos considerar que el cartílago tiene que ser bien obtenido y bien tallado, con suficiente proyección. Hay que localizar bien la posición de la nueva oreja de acuerdo a los parámetros antropométricos delineados y por último disecar adecuadamente con especial cuidado la circulación de la cubierta cutánea para fijar los fundamentos sobre los cuales seguir edificando los otros tiempos de la reconstrucción de la oreja y llevar a feliz término el proceso, obteniendo resultados uniformes y permanentes en todos los casos de pérdida de pabellón auricular, ya sea de etiología congénita, traumática o quirúrgica, aún en los casos en que se ha visto dañada la piel del área mastoidea.

\section{Dirección del autor}

Dr. Aníbal I. Mantilla M.

Calle $119^{\mathrm{a}} \mathrm{N}^{\mathrm{o}} 7-35$

Bogotá - Colombia

e-mail: aesculapio@msn.com

\section{Bibliografía}

1. Gillies,.H- Millard, R.: "The Principles and Art of Plastic Surgery". Vol.I. Boston- Toronto. Little Brown and Company, 1957, Pp.302-309.

2. Mc. Carthy J.G.: "Cirugía Plástica". Vol. II. Buenos Aires. Editorial Médica Panamericana. 1992. Pp.1198 - 1213.

3. Grabb. W. Smith: "Plastic Surgery", Third edition.-Little Brown and Co. Boston.-1979. Pp 299 - 317.

4. Brent.B.: "A personal approach to total auricular construction: Case Study".- Clin. Plast. Surg. 1981, 8 (2):211.

5. Lesavoy M..A.: "Reconstruction of the Head and Neck" Williams and Wilkins Co. Baltimore/London, 1981 Pp.90.

6. Brent B.: "Technical Advances in ear reconstruction with autogenous rib cartilage grafts; personal experience with 1.200 cases". J.of Plast. Rconstr. Surg. 1999, 104 (2). 